Results.-Cured, 9 ; relieved, 12 ; irregular, 16.

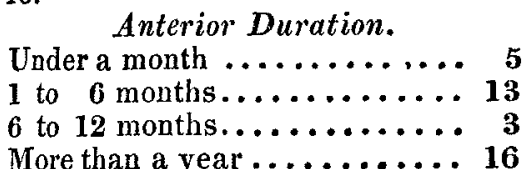

Treatment.-Careful diet, gentle laxatives, alkalies, opiates, and the nitrate of silver, were the principal means employed.

The chief causes were improper food and mental anxiety. The sympathetic disturbances principally affected the brain, heart, and colon. The atonic and nervous forms of dyspepsia prevail most among the poor.

GASTRO-ENTERITIS.

Sex.-Males, 19 ; females, 20.

$$
\text { Ages. }
$$

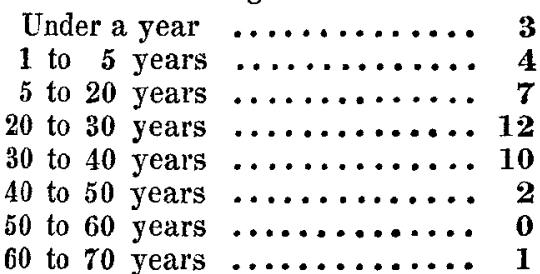

The influence of sex seems to be negative. The greater liability of the middle period of life is still evident.

\section{Anterior Duration.}

Under a month ........... 16

From 1 to 3 months....... 10 3 to 6 months....... 5

6 to 12 months....... 6

More than a year .......... 2

Result.-Cured, 21 ; relieved, 6 ; irreguIar, 12.

Treatment.-Acute cases in general are easily sublued, but chronic cases are perhaps among the most obstinate and difficult. This applies particularly to the poor. Dr. Baillie's combination of cusparia, nitric acid, and laudanum, proved useful in three obstinate cases. The sesquioxide of iron with port wine answered well where anemia was combined, and the sulphate of copper and nitrate of silver with opium succeeded in several instances. We had also reason to think well of burnt rhubarb, and have combined it advantageously with steel in two very delicate cases.

\section{DIARRHCA.}

This term includes cases with increased secretion, but without the symptoms of local inflammation, and with little or no febrile disturbance. The majority occurred during the autumn, and several followed measles.

Sex.-18 males; 24 females.

$$
\text { Ages. }
$$

Under 3 years ............ 14

From 3 to 10 years $\ldots \ldots \ldots .4$ 10 to 20 years ....... 8 20 to 30 years .......6

No. 981 .

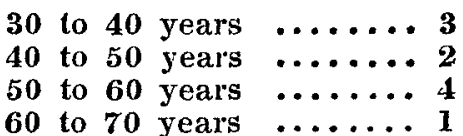

Anterior Duration.

Under a month ............ 26

From 1 to 3 months....... 5

3 to 12 months....... 6

More than a year.......... 3

Not registered ............ 2

Result._Cured, 32 ; irregular, 10.

Average duration of treatment in 18 cases, nine days.

Treatment.-20 were successfully treated by the chalk mixture with ammonia and opium, preceded in a few instances by a mild mercurial. In eight chronic cases much benefit resulted from the sesquioxide of iron in port wine. The sulphate of copper and opium were successful in two cases: the decoction of logwood and chalk mixture succeeded in one instance. Lime water, the warm bath, strict farinaceous diet, and flannel clothing, were the main points in the general treatment. In cases of chronic mucous membrane disease occurring in children, characterised by variable and often craving appetite, lienteric diarrhoea, emaciation, and pyrexia, we have seen much benefit from the one-sixteenth to one-fourth of a grain of calomel and quinine, from three to six times a-day, and combined with a milk and flour diet, an occasional laxative of castoroil and the warm bath. Some cases considered hopeless have recovered under these means.

(To be continued.)

\section{STATISTICAL}

\section{REGISTER OF DISEASE}

IN THE

\section{WESTMINSTER HOSPITAL.}

\section{To the Editor of THe LANCET.}

Sir,-During my residence of three yoars and a half at the Westminster Hospital, I have been in the habit of Ikeeping a statistical record of the cases admitted into that institution, which I have found not only useful on many occasions, as a source for reference, but interesting and instructive from the mass of information gradually accumulated.

Should the following abstract from that register be considered worthy a place in your periodical, you will oblige me by its insertion when an opportunity occurs.

$I$ have to a certain extent followed the arrangement adopted by Dr. Macleod, in a report, which he published a few years ago, of the cases admitted under his care in St. George's Hospital, but which, I believe, he 
has of late discontinued. I am, Sir, your obedient servant,

J. H. Baller, M.R.C.S.,

Resident Medical Officer at the

June 2, 1842.

Westminster Hospital.

Anstract from the Statistical Register of Patients admitted and discharged during the Year 1841, comprising also the Sex, Age, Disease, Result, and immediate Cause of Death.

$$
\begin{array}{rr}
\text { Admitted-Males...... } & \mathbf{6 8 4} \\
\text { Females... } & \mathbf{6 4 8} \\
\text { Total.. } & \mathbf{1 3 3 2}
\end{array}
$$

Males. Females.

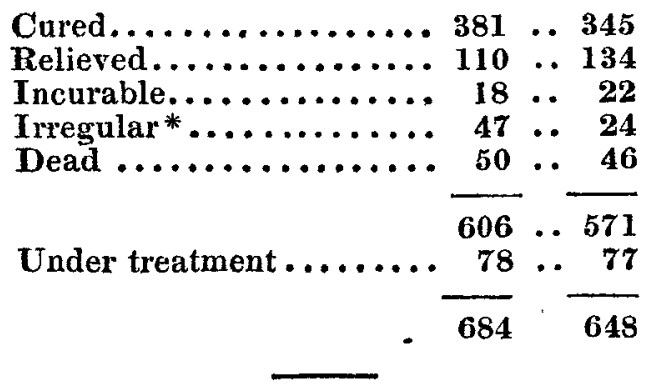

CLASSIFICATION OF DISEASES.

Cerebral Diseases-No. 45.

Apoplexia, 7 ; deąd, 1.

Hemiplegia, 4.

Epilepsia, 8; dead, 1.

Phrenitis, 1; dead, 1.

Cerebral congestion, 1 .

Cephalæa, 5.

Hydrocephalus, 2 ; dead, 2.

Delirium tremens, 3 ; dead, 2.

Tumor cerebri, 3 ; dead, 2.

Various organic affections of the encephalon, 5.

Diseases of the Nervous System-No. 30.

Hysteria, 19.

Hypochondriasis, 4.

Neuralgia, 4.

- Chorea, 3.

Affections of Fauces-No. 17.

Tonsilitis, 13.

Ulcer of throat, 4.

Otalgia, 2.

Affections of Respiratory Organs-No. 135.

Laryngitis, 4 ; dead, 1.

Broncho-pneumonia, 16 ; dead, 6 .

Pleuro-pneumonia, 6 ; dead, 2.

* This includes those discharged for irregularity of conduct, or refusing to follow the directions of the medical officers.
Bronchitis, 38 ; dead, 4.

Phthisis, 54; dead, 17.

Catarihus, 8.

Pleuritis, 3.

Empyema, 1.

Pertussis, 2.

Pulmonary apoplexy, 2 ; dead, 1.

Gangræna pulmonum, 1; dead, 1.

Organic Affections of Heart, \&c, -No. 20,

With dropsy, 4 ; dead, 1.

Without dropsy, 11.

Pericarditis, 2 ; dead, 1.

Diseased arteries, 2.

Aneurism of aorta, 1 ; dead, 1 .

Various Diseases of Abdominal VisceraNo. 98.

Various forms of dyspepsia, 28.

Gastritis (subacute), 4.

Hæmatemesis (vicarious), 5 .

Ditto (à morbo jecoris), 2.

Scirrhus pylori, 2 ; dead, 2.

(One being complicated with ma. lignant tuber of the liver.)

Enteritis, 1 ; dead, 1.

Hæmorrhœa, 1.

Gastro-enteritis, 1.

Diarrhoa, 5.

Colica (accidentalis), 8.

Ditto (à plumbo), 7 .

Hepatitis (chronic), 8; dead 1.

Ditto (with dropsy), 8; dead, 1 .

Ditto (acute), terminating in abscess, 1 ; dead, 1.

Icterus, 5.

Peritonitis (chronic), 4 ; dead, 2.

Ditto (post partum), 1.

Mesenteric disease, 1 ; dead, 1.

Tumor abdominis, 1.

Disease of colon, 1 .

Disease of rectum, 1 .

Diseased kidneys, with dropsy, 14; dead, 5.

Dropsy (after scarlatina), 1.

Renal calculi, 7.

Vesical calculus, 1.

Diseased prostate, 2.

Incontinence of urine, 3.

Retention of urine, $\mathbf{3}$.

Stricture, 10.

Diseases of the Uterine System-No, 89

Amenorrhoea and chlorosis, 45 .

Dysmenorrhoea, 5 .

Leucorrhoea, 10.

Menorrhagia, 7 .

Uterine hæmorrhage, 4 .

Tumor uteri, 4.

Procidentia uteri, 7 .

Ovarian dropsy, 1 ; dead, 1.

Malignant disease of ovary, 2 ; dead,2.

Cancer uteri, 2.

Ditto pudendi, 1 ; dea 1 .

Corroding ulcer of uterus, 1 ; dead, 1 . 
Diseases of Muscular and Fibrous Tissues. Rheumatism (acute), 30.

Ditto (subacute and chronic), 90. Synovial inflammation of knee-joiut, 30 . Ditto ditto of bursaat knee, 6 . Ditto ditto of shoulder-joint, 2. Periostitis, 2.

Febris (adynamica), 13 ; dead, 4.

Ditto (continua), 28.

Ditto (intermittens), 1.

Erysipelas (traumatic), 15 ; dead, 7.

Ditto (idiopathic), 4 ; dead, 1.

Phlegmon, 3.

Tumores, 7 .

Scrofulous diseases, 17 ; dead, 1.

Inflamed absorbents, 5 .

Gangræna senilis, 3 ; dead, 2.

Abscess, 28.

Ulcers, $\mathbf{7 4}$.

Cutaneous Diseases-No. 27.

$\begin{array}{ll}\text { Lepra, 4. } & \text { Psoriasis, 2. } \\ \text { Eczema, 7. } & \text { Impetigo, 2. } \\ \text { Porrigo, 4. } & \text { Lichen, 1. } \\ \text { Prurigo, 2. } & \text { Rupia, 4. } \\ \text { Lupus, 1. } & \end{array}$

Scarlatina, 1 .

Anæmia, 5.

Cachexia, 4.

Rachitis, 2.

Tænia, 1.

Poison by nux vomica, 1.

Syphilis, 19.

Secondary syphilis, 22.

Gonorrhœea, \&c., 37.

Fistula in ano, 3.

Ditto, in perinæo, 2.

Recto-vaginal fistula, 1.

Hæmorrhoids, 5.

Cancer scroti, 1 ,

Osteo-sarcoma, 1 ; dead, 1.

Fungoid disease, 1 ; dead, 1.

Anthrax, 1.

Burns and scalds, 14 ; dead, 5.

Diseases of eye and appendages, 21.

Various diseases which cannot well be arranged under any class, 16.

Simple fractures of leg (men), 18.

(One dead from erysipelas.)

Compound fractures of leg (men), 4.

(One died on the third day from gangrene.)

Fractured arm (men), 3.

Ditto cranium (men), 2 ; dead, 1.

Ditto ribs (men), 6 .

Simple fractures of leg (women), 5 .

Fractured arm (women), 3.

Fractured ribs (women), 2.

Dislocations : hip, 1 ; shoulder, 1.

Diseases of bone, 9 .

Diseases of joints (including hip, 12), 23; dead, 2.

Hernia, strangulated femoral (operation), $\mathbf{1}$.

Hernia (reduced), 2.

Tetanus (traumatic), 1 ; dead, 1.

Contractions from burns, 2 .
Club-foot, 2.

Cut-throat, 1.

Accidents, \&c., contusions, sprains, 204 ; dead, 5 .

Abstract showing the Diseases, \&c., which caused Death, and the several Ages of the Patients.

Apoplexia, 1: age 60 years.

Epilepsia, 1 : age 23.

Phrenitis, 1 : age 25.

Hydrocephalus, 2 : ages 5, 6.

Delirium tremens, 2 : ages $40,50$.

Tumor cerebri, 2 : ages 19,47 .

Laryngitis, 1: age 54 .

Broncho-pneumonia, 6 : ages 31, 40, 40, $41,53,54$.

Pleuro-pneumonia, 2 : ages 7, 23.

Bronchitis, 4: ages 29, 45, 55, 68 .

Phthisis-males, 10 ; females, 7 ; total, 17 : ages 18, 19, 22, 23, 24, 27, 28, 30, 32, $32,34,42,50,50,54,60,66$.

Gangræna pulmonum, 1 : age 55.

Pulmonary apoplexy, 1 : age 61 .

Organic affection of heart, 1 : age 51.

Pericarditis, 1 : age 34.

Aneurism of aorta, 1 : age 39.

Scirrhus pylori, 2 (both females): ages 43,45 . (In one it was complicated with malignant taber of the liver.)

Organic disease of liver, with dropsy, 1 : age 35. Ditto, without dropsy, 1 : age 31.

Acute inflammation of liver terminating in abscess, 1 : age 21.

Chronic peritonitis (tubercular), 2 : ages 17 each.

Enteritis, 1 : age 34.

Mesenteric disease, 1 : age $3 \frac{1}{2}$.

Dropsy with diseased kidney, 5 : ages 11, $30,52,57,60$.

Malignant disease of ovary, 2: ages 30, 50.

Ovarian dropsy, 1 : age 52.

Cancer pudendi, 1 : age 50

Corroding ulcer of womb, 1 : age 30 .

Adynamic fever, 4 : ages 21, 35, 48, 58 .

Erysipelas (traumatic), 5: ages 21, 25, $38,58,78$. (Three of these patients were attacked with the disease whilst in the hospital.)

Ditto (idiopathic), 1 : age 23.

Phlegmonous erysipelas, 2: ages 47, 63.

Medullary sarcoma, 1 : age 24 .

Gangræna senilis, 2: ages 70, 79.

Diseased spine, 1: age 19.

Tetanus (traumatic), 1 : age $\mathbf{3 2}$.

Osteo-sarcoma of lower jaw, 1 : age 62 .

Scrofulous disease of shoulder-joint, 1 : age 65 .

Diseased hip-joint, 3 : ages 7, 7, 48 .

Burns and scalds, 5 : ages $2,2,3,4,4$.

Fractured skull, 1: age 15.

Accidents, fatal, 5 : ages $9,33,40,70,79$.

Total number of deaths during the year $1841-96$. 
Table showing the various Ages of the Patients admitted during the Year 1841, and the number of Deaths in each Decennial Period.

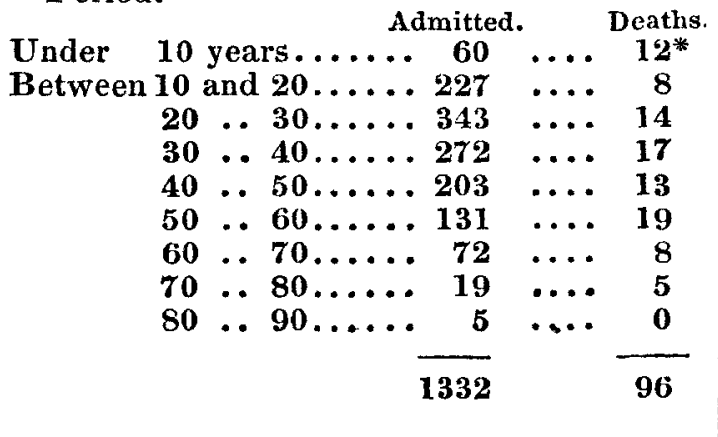

ON THE

PREVALENCE OF ALMOST UNCONSCIOUS PARTURITION IN

MANUFAC'TURING DISTRICTS;

WITH REMARKS ON UNCONSCIOUS CONCEPTION AND PARTURITION.

By Charles Clay, M.D., M.R.C.S., \&c., Manchester.

I HAVE very frequently had occasion to observe, in a long practice amidst a factoryworking population, the great facility with which many females get through their labours in some cases; this is to such an extent, and accompanied with so little emotion or apparent suffering on the part of the patient, as to border on unconsciousness, at least extreme apathy to the circumstances connected with and surrounding them at the time. Even in first cases I have seen a sort of hardy, if not heroic, determination (or rather, I might say, dogged stupidity,) not to move a limb, or express the slightest feeling of pain during labour.

The cases most remarkable for this are those who have had perhaps three or four children previously, and are of a pale, exsanguineous complexion, of a lax, muscular fibre, and rather above than below the average stature; the too common features of factory employment. Under such circum. stances $I$ have seen cases where it was next to impossible to say if the patient was labouring under the effects of uterine pains and contraction, had it not been for the pressure of the uterine mass against the finger during an examination. I attribute this peculiarity, in a great measure, to the debilitating nature of their employment, prematurely maturing the system, and by the high temperature of the mills, some of which run up from 85 to 90 Fahrenheit, producing a continuous and too copious perspiration,

* Of the deaths under 10 years, 5 were the result of burns and scalds. creating that system of muscular laxity which accounts for the great facility of many of their labour's ; to which may be added, the early time of life many of them become mo. thers (having had three instances of their being mothers at fifteen years of age), and the children (at birth) being somewhat less than the average weight and size.

Still, with all these debilitating facilities, I have never as yet witnessed a case (that is, of sound mind, and under the average circumstances of bodily health,) that could be said to be unconscious of delivery. That some will from known, others from causes unknown, doggedness, or spirit of bravado, attempt to conceal the common feelings observed on such occasions, I allow; and that they frequently carry this to an almost incredible extent: still $\mathbf{I}$ maintain, with most medico-legal writers, that we have yet to see unconscious delivery proved, as well as that of unconscious conception, excepting in both cases under the influence of narcotics, idiotcy, or coma, from disease. And that unconscious conception and delivery are incompatible with a sound state of mind and healthy condition of body, I am led to make these remarks in consequence of a communication in The LanceT of November 27, 1841, page $\mathbf{2 9 4}$.

The following circumstances are stated, unfortunately, too briefiy for a case which the relater observes to be one of the utmostim. portance in a medico-legal point of view. The case is as follows, copied from THR LANCET, communicated by T. E. Rawson, Esq., of Leicestershire:- "I was sent for to attend a young married woman in her first confinement. The message was sent after the waters had been unexpectedly discharged, I had to ride a distance of three miles, but found she had had no pains, and she assured me the discharge of waters was not attended with any uneasiness, not even sufficient to have awaked her had she been asleep at the time. On examination, I found the os uteri dilated, and the head presenting. The child was slowly and unintermittingly but forcibly expelled. She betrayed no symptoms of uneasiness whatever, and though I uatched her countenance she did not exhibit the least consciousness of the child's expulsion, but expressed her surprise on seeing it. The child was strong and lively, and with the mother did well. The mother was about twenty-two years of age, short, plethoric, and healthy. She was strictly respectable, and had been married full ten months; indeed she could have no motive whatever for disguising her feelings.

"I conceive the above case is interesting" in a medico-legal point of view. Dr. Montgomery doubts the possibility of such an occurrence 'excepting under peculiar circumstances, certainly not in a first delivery.' In Beck's Jurisprudence it is said 'that the possibility of a woman being delivered with- 